Flow (PEF) is usually the most convenient first step in the confirmatory process. We previously described a statistical method of analysis comparing mean 2-h values on work and rest days which required the worker to wake at similar times on rest and work days. This was achieved in only $43 \%$ of records. We describe a new method of timepoint analysis without this restriction and overcoming a theoretical problem with the original analysis (the assumption that the variance of the waking reading was the same as the variance at other times of the day).

Methods Workers were asked to measure PEF approximately 2-h from waking to sleeping for 3-4 weeks. 236 PEF records from workers with independently diagnosed occupational asthma, and 320 from asthmatic controls were available. Readings were grouped by the time since waking, in an attempt to correct for changes in diurnal variation induced by changes in shift and waking time. Daily PEF measurements were meaned into matching 2-h time segments. The pooled SD for rest day measurements (excluding waking readings) was obtained from a one-way ANOVA. Timepoints with mean workday PEF statistically lower (at the Bonferroni adjusted 5\% level) than the restdays were counted, after adjusting for the number of contributing measurements at each point.

Results A minimum of four analysable timepoint comparisons per day was needed. $78 \%$ of records were suitable for analysis. Records with one or more timepoints statistically worse on workdays gave a sensitivity of $71 \%$ against independently diagnosed occupational asthma and a specificity of $93 \%$ in non-occupational asthmatics.

Conclusion The removal the requirement to wake at similar times on work and rest days increased the utility of timepoint analysis for the diagnosis of occupational asthma from 43-78\% without compromising sensitivity or specificity. Statistical validity was also improved.

\section{P10 ASBESTOS-INDUCED DIFFUSE PLEURAL THICKENING-A CONTINUING PROBLEM}

doi:10.1136/thx.2010.150961.10

V Jeebun, SC Stenton. Regional Unit for Occupational Lung Diseases, Royal Victoria Infirmary, Newcastle Upon Tyne, UK

Introduction Asbestos-induced diffuse pleural thickening (DPT) remains a relatively common but poorly understood disease.

Methods We reviewed the clinical, physiological and radiological features of patients referred to our department for assessment. Diagnosis was based on a history of asbestos exposure, chest radiographic pleural thickening with blunting of costophrenic angle, and exclusion of other likely causes of pleural disease.

Results 75 patients were identified. All were male. Mean age was $65 \pm 9$ years. Asbestos exposures occurred in shipyards $(n=35)$, construction work $(n=19)$, power stations $(n=4)$ and other/multiple sites $(\mathrm{n}=17)$. Median duration of asbestos exposure was 13 years. Presentation occurred at a median of 36 years (range: 12-55years) after onset of exposure. Pleural disease was an incidental radiological finding in $18 \%(n=14) .72 \%$ presented with breathlessness, $27 \%$ with chest pain, and $11 \%$ had flu-like symptoms. $40 \%(n=30)$ presented with a pleural effusion, which was suspected to be asbestos-related. Mean latency for development of pleural effusions was 31 years (range: $12-55$ years). 24 of these were unilateral only, and 6 were bilateral. Right-sided effusions were five times more prevalent than left-sided effusions. After the diagnosis of a pleural effusion, DPT was noted radiologically after a median of 7 months (range: 1 month to 2 years). In $10 \%(n=3)$, the effusion persisted over a median follow-up period of 2 years. Overall, $73 \%(n=55)$ had unilateral disease at presentation and $24 \%(n=13)$ were observed to progress from unilateral to bilateral disease after a mean time of $3.1 \pm 3.6$ years (range: 1 month to 13 years) after onset of disease or diagnosis. Once established, the degree of thickening remained stable in $91 \%$ $(n=68)$. There were no significant differences in either duration of asbestos exposures or latency in patients with stable versus progressive disease. No difference in duration of asbestos exposures was also found between those with unilateral versus bilateral disease. Most patients had restrictive abnormalities on lung function testing with mean TLC $74 \%$ and mean RV $73 \%$ of predicted. Radiographic appearances correlated poorly with lung function impairment or symptom progression.

Conclusion Understanding its natural history should help clinicians diagnose and manage asbestos-induced benign pleural thickening.

\section{P11 CARDIO-RESPIRATORY FITNESS AT WORK; THE EFFECTS OF PUBLIC HEALTH GUIDANCE?}

doi:10.1136/thx.2010.150961.11

M Naughton, JSS Gaynor, L Bradshaw, A Curran, S Till, D Fishwick. Centre for Workplace Health, HSL, Buxton, UK

Physical activity is important to improve and maintain cardiorespiratory fitness. More recently, additional wider health benefits of physical exercise have been highlighted, including reduction in obesity and promoting mental wellbeing. Increased recognition of these benefits has led NICE to issue recent public health guidance in this area ( $\mathrm{PH} 13$; promoting physical activity in the workplace). These recommendations aim to help employers and workplace health professionals prevent diseases associated with a lack of physical activity. We have audited the approach taken to physical activity at work taken by two large organisations, using PH13 to develop 23 appropriate gold standards (GS). For example, GS1 states that 'There is an organisational policy to encourage and support employees to be more physically active', GS5 that 'There are organisational goals for physical activity in the workforce' and GS10 that 'There are policies to encourage employees to be physically active while travelling to work (eg, walking and cycling)'. 44 employees from a health provider (Health) and 63 employees from a public sector organisation (PSO) underwent a self-completed questionnaire enquiring about a broad range of workplace-based exercise issues, each question mapping to an a priori defined gold standard. Various clear differences emerged between organisations in attaining the GS, although compliance by both with certain of these was good. For example, GS7 stated 'A multi-component programme is in place to encourage and support employees to exercise'. Overall, employees from Health and PSO were positive about the facilities to encourage exercise, the majority of employees being aware of various facilities in place including safe bike storage (PSO; $73.0 \%$, Health 52.3\%) and showers (PSO; 87.3\%, Health; 40.9\%). Compliance with other GS was poor. For example, GS9 required that there was employee knowledge of an incentive schemes to encourage exercise'. Despite the presence of incentives to exercise at each site, only $14.3 \%$ of PSO employees (Health; $13.6 \%$ ) were aware of financial incentives and 9.5\% (Health; 9.1\%) aware of non-financial incentives to exercise. Whilst exercise is an important contributor to health, further work is required to ensure that recent $\mathrm{PH}$ guidance aimed at improving exercise at work has maximal worker benefit.

\section{P12 ALARMING IGNORANCE ABOUT THE DANGERS OF ASBESTOS AMONG UK HOMEOWNERS}

doi:10.1136/thx.2010.150961.12

N Eiser, I Jarrold, H Richardson, K Huntly. British Lung Foundation, London, UK

Introduction Nearly 14 million homes were built possibly using asbestos-containing construction materials between 1950 and the mid-1980s. Certain types of asbestos were not banned until 1999. 